ARTICLE

DOI: $10.1038 / s 41467-018-04946-7$

\title{
Excited-state vibrational dynamics toward the polaron in methylammonium lead iodide perovskite
}

\author{
Myeongkee Park ${ }^{1,2}$, Amanda J. Neukirch ${ }^{3}$, Sebastian E. Reyes-Lillo4,5,6, Minliang Lai ${ }^{1}$, Scott R. Ellis ${ }^{1}$, \\ Daniel Dietze (1) ${ }^{7}$, Jeffrey B. Neaton ${ }^{5,6,8}$, Peidong Yang ${ }^{1,8,9,10}$, Sergei Tretiak (1) ${ }^{3}$ \& Richard A. Mathies (1) ${ }^{1}$
}

Hybrid organic-inorganic perovskites have attractive optoelectronic properties including exceptional solar cell performance. The improved properties of perovskites have been attributed to polaronic effects involving stabilization of localized charge character by structural deformations and polarizations. Here we examine the $\mathrm{Pb}-\mathrm{I}$ structural dynamics leading to polaron formation in methylammonium lead iodide perovskite by transient absorption, time-domain Raman spectroscopy, and density functional theory. Methylammonium lead iodide perovskite exhibits excited-state coherent nuclear wave packets oscillating at $\sim 20, \sim 43$, and $\sim 75 \mathrm{~cm}^{-1}$ which involve skeletal bending, in-plane bending, and c-axis stretching of the $\mathrm{I}-\mathrm{Pb}-\mathrm{I}$ bonds, respectively. The amplitudes of these wave packet motions report on the magnitude of the excited-state structural changes, in particular, the formation of a bent and elongated octahedral $\mathrm{Pbl}_{6}{ }^{4-}$ geometry. We have predicted the excited-state geometry and structural changes between the neutral and polaron states using a normal-mode projection method, which supports and rationalizes the experimental results. This study reveals the polaron formation via nuclear dynamics that may be important for efficient charge separation.

\footnotetext{
${ }^{1}$ Department of Chemistry, University of California, Berkeley, CA 94720, USA. ${ }^{2}$ Department of Chemistry, Dong-A University, Busan 49315, Republic of Korea. ${ }^{3}$ Theoretical Physics and Chemistry of Materials, Los Alamos National Laboratory, Los Alamos, NM 87545, USA. ${ }^{4}$ Departamento de Ciencias Físicas, Universidad Andres Bello, Santiago 837-0136, Chile. ${ }^{5}$ Department of Physics, University of California, Berkeley, CA 94720, USA. ${ }^{6}$ Molecular Foundry, Lawrence Berkeley National Laboratory, Berkeley, CA 94720, USA. ${ }^{7}$ Osram Opto Semiconductors GmbH, Leibnizstraße 4, Regensburg 93055, Germany. ${ }^{8}$ Kavli Energy NanoSciences Institute at Berkeley, Berkeley, CA 94720, USA. ${ }^{9}$ Department of Materials Science and Engineering, University of California, Berkeley, CA 94720, USA. ${ }^{10}$ Materials Sciences Division, Lawrence Berkeley National Laboratory, Berkeley, CA 94720, USA. Correspondence and requests for materials should be addressed to R.A.M. (email: ramathies@berkeley.edu)
} 
ybrid organic-inorganic perovskites (HOIP) composed of an organic cation and inorganic framework are one of the most promising new solar cell materials ${ }^{1-10}$. These materials are exciting because of their high power conversion efficiency (PCE) as well as their low-cost and facile fabrication ${ }^{11}$. Their efficiency has reached $\sim 23 \%$ which is now comparable with single crystalline silicon-based solar cells ${ }^{12}$. However, understanding the underlying mechanisms of this high PCE is still a significant challenge.

Polycrystalline HOIP fabricated at low temperatures $\left(\sim 100^{\circ} \mathrm{C}\right)$ has significant crystalline inhomogeneity: defects and grain boundaries typically introduce multiple energy-loss trap sites that may significantly lower the $\mathrm{PCE}^{13-20}$. This inhomogeneity is surprising because most other inorganic solar cell materials require pure crystalline composition for high $\mathrm{PCEs}^{21,22}$. Altogether these observations suggest that the inhomogeneity in the polycrystalline HOIP does not greatly influence the performance by protecting free charge carriers from ultrafast thermal relaxation, although these defects do accumulate in humidity-degraded materials ${ }^{23-25}$. Associated with the high PCE, polycrystalline HOIP exhibits a long lifetime $(\tau \sim 1-3 \mu \mathrm{s})$ and a long diffusion length $\left(L_{\mathrm{D}} \sim 1-3 \mu \mathrm{m}\right)$, as well as modest mobility $\left(\boldsymbol{\mu} \sim 30-100 \mathrm{~cm}^{2}\right.$ $\left.\mathrm{V}^{-1} \mathrm{~S}^{-1}\right)$ for the free charge-carriers ${ }^{26-28}$. These characteristics indicate the efficient suppression of unfavorable ultrafast chargerecombination and suggest that unique excited-state dynamics may enable the longer charge-carrier lifetime and their impunity to thermal relaxation ${ }^{29}$.

The formation of a polaron, a charged quasi-particle, may account for the unique excited-state photo-physical properties in $\mathrm{HOIP}^{30,31}$. In principle, the charge-localized polaron state is energetically unstable without further stabilization. Once it is formed in a higher energy state, it should quickly relax via high energy phonon scattering ${ }^{32}$. However, polaron formation in HOIP can be favorable; electric dipole reorientation of the nearby organic cations not only minimizes the Gibbs free energy of the polaron but also protects the charges from other ultrafast thermal relaxations according to Zhu and coworkers ${ }^{29,32,33}$. They have successfully measured femtosecond time-resolved fluorescence of the hot electron-polaron in methylammonium lead iodide $\left(\mathrm{MAPbI}_{3}\right)$ and shown that after a few hundred femtosecond relaxation, which is related to the methylammonium (MA) cation reorientation time, the polaron lives as long as $\sim 100 \mathrm{ps}^{24}$.

We have recently reported a theoretical prediction on the structure of the small electron polaron of $\mathrm{MAPbI}_{3}$, which exhibits not only MA dipole reorientations toward the charge but also the structural distortion of the inorganic $\mathrm{Pb}-\mathrm{I}$ framework to stabilize the isolated charge ${ }^{34}$. In particular, the structural distortion associated with a polaron suggests that ground and excited-state potential energy surfaces (PES) have different energy minima along nuclear coordinates that are related to the distortion. Thus, coupled electron-phonon dynamics can be coherently excited on the polaron PES by impulsive excitation, where the associated reorganization energies $(\lambda)$ and displacements $(\Delta)$ determine the polaron formation pathways ${ }^{35,36}$. These coherent dynamics have been detected by using femtosecond time-resolved spectroscopies $^{37,38}$. For example, Wang et al. ${ }^{39}$ recently observed a single coherent wave packet oscillating at $\sim 23 \mathrm{~cm}^{-1}$ in a $\mathrm{MAPbI}_{3}$ film using femtosecond transient absorption. However, additional experimental studies of excited-state dynamics in HOIP are necessary to resolve higher frequency vibrations disclosing the structural dynamics of polaron formation.

Herein, we report the production and analysis of coherent nuclear wave packets in $\mathrm{MAPbI}_{3}$ that oscillate at low frequencies $\left(<100 \mathrm{~cm}^{-1}\right)$ as measured by femtosecond impulsive stimulatedRaman spectroscopy (ISRS). We also present first principles density functional theory (DFT) simulations of the structural distortions associated with polaron state. The $\mathrm{PbI}_{6}{ }^{4-}$ octahedral charge-state geometry changes based on the experimental excited-state Raman displacements and the theoretical simulation are then compared. These results reveal coherent $\mathrm{Pb}-\mathrm{I}$ vibrational normal mode dynamics that lead to the stabilized polaron state.

\section{Results}

Coherent wave packet measurement and analysis. We probed the transient excited-state absorption (ESA) band of $\mathrm{MAPbI}_{3}$ perovskite in the near infrared (NIR) wavelength $(830-940 \mathrm{~nm})$ region which is distinct from the ground-state absorption (GSA), stimulated emission (SE) and other bleach bands (see Methods). Fig. 1a presents the contour plot of the ESA band centered at $\sim 855 \mathrm{~nm}$. There are no dynamic peak growths or shifts after the response-limited appearance; the ESA simply shows an exponential intensity decay in the observation window. Three representative time-profiles in the 840-855, 860-875, and 885-900 nm region are presented in Fig. $1 \mathrm{~b}-\mathrm{d}$. The rise times are all resolution limited and each decay is well fit by a biexponential decay with constants of $\sim 240$ fs and $3.8-5.1$ ps. Especially, the fits to the red and blue traces suggest that these data also contain underlying temporal oscillations. The difference residuals between the exponential fits and the experimental data in Fig. 1 confirm the existence of these temporal oscillatory components that are likely due to coherent nuclear wave packet dynamics.

Fast Fourier transform (FFT) and linear prediction with singular value decomposition (LPSVD) analyses were performed to quantitate the oscillatory components in the NIR ESA band. The FFT spectra are presented from the residuals extracted from the exponential fittings at each wavelength (Fig. 2a), which mostly exhibit Raman modes lower than $150 \mathrm{~cm}^{-1}$. The FFT spectral intensities exhibit a bi-lobed pattern with strong components below $860 \mathrm{~nm}$ and above $880 \mathrm{~nm}$ but weak amplitudes around $865 \mathrm{~nm}$ near the ESA maximum (Supplementary Fig. 1). This result is consistent with the expectation that when a spectral band position is oscillating in time, the largest signal differences are on the red and blue sides, where the slope is highest and the lowest amplitude oscillations are near the peak center ${ }^{40,41}$. Figure $2 b, c$ presents FFT and LPSVD results extracted in the indicated blue and red regions, which both exhibit common low frequency modes at $\sim 20, \sim 43$, and $\sim 75 \mathrm{~cm}^{-1}$, supporting our wave packet analysis. In addition, the FFT spectra reveal very small 110 and $150 \mathrm{~cm}^{-1}$ Raman peaks which were also found in our previous resonance Raman studies ${ }^{42}$, but we were unable to perform a more detailed analysis of the latter bands here because of their weak intensities.

To examine whether these oscillatory signals are indeed due to band position oscillation of the ESA band we examined the relative phase of these oscillations. In principle, for the band position shift model they should be $\sim 180^{\circ}$ out of phase. However, it is sometimes difficult to extract the ideal phases and frequencies when there are highly damped and mixed oscillations with other frequencies. This ambiguity may result in serious overestimation using LPSVD analysis. In order to avoid this error, we crosschecked both the FFT and LPSVD results, as shown in Fig. 2. Figure $3 \mathrm{~d}$ presents the phase parameters for the $\sim 20, \sim 43$, and $\sim 75 \mathrm{~cm}^{-1}$ modes from the LPSVD results. The most intense wave packet at $\sim 20 \mathrm{~cm}^{-1}$ has the largest phase shift from $150^{\circ}$ to $0^{\circ}$ which is nearly out of phase as expected. The 45 and $75 \mathrm{~cm}^{-1}$ modes exhibit relative phases of $240 / 170$ and $140 / 80$ on the red/ blue sides that are roughly $60-70^{\circ}$ out of phase. The deviation from the expected $180^{\circ}$ phase differences might result from the somewhat larger errors in these phase determinations than for the $20 \mathrm{~cm}^{-1}$ mode. Especially, the $\sim 180^{\circ}$ out of phase features of the strongest $\sim 20 \mathrm{~cm}^{-1}$ mode together with the amplitude pattern in 
a

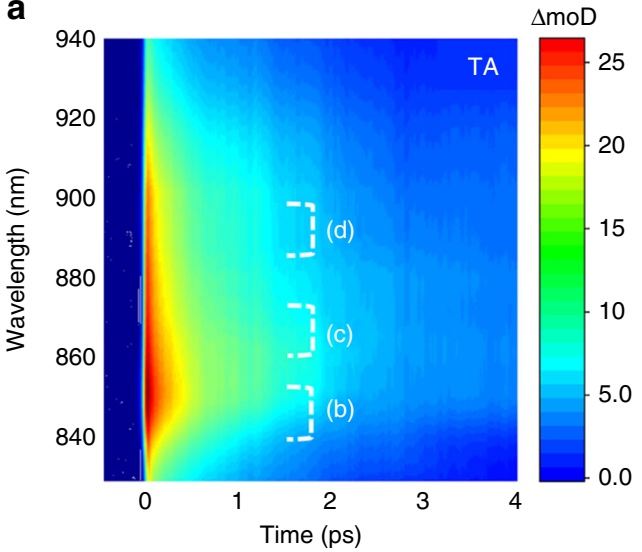

b

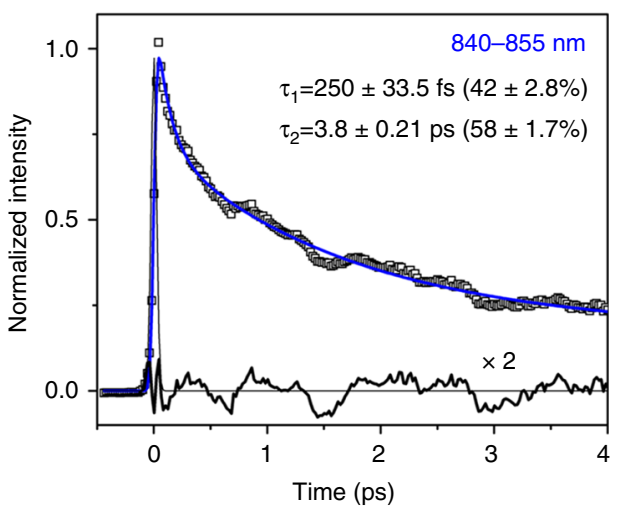

C

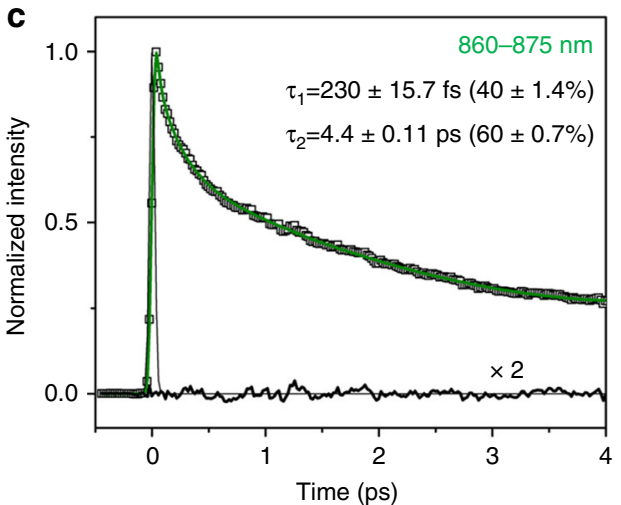

d

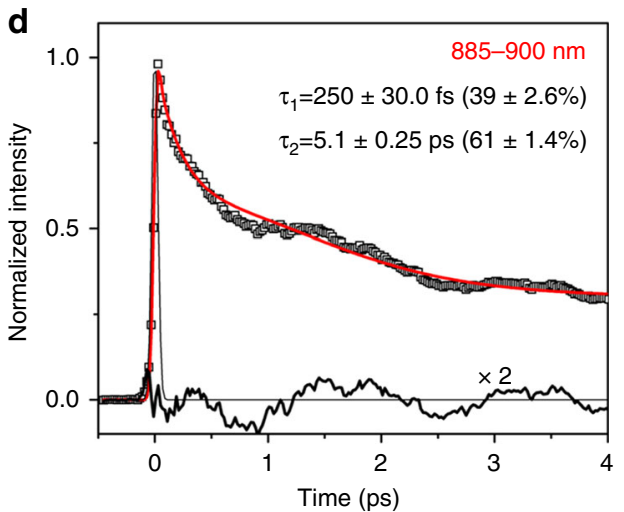

Fig. 1 Transient excited state absorption spectrum shown in the $830-940 \mathrm{~nm}$ region of MAPbl 3 perovskite excited by a $\sim 40$ fs FWHM pulse at 560 nm. a Contour plot of excited state absorption band centered at $~ 855 \mathrm{~nm}$. b-d Time profiles measured at $840-855$ (blue), $860-875$ (green), and $885-900 \mathrm{~nm}$ (red) fit to the sum of two exponentials with the indicated constants. The residual profiles are also shown revealing underlying oscillations especially in the $840-855$ and $885-900 \mathrm{~nm}$ regions

Fig. 2a, support the idea that these signals are due to coherent nuclear wave packets which are propagating on the excited-state PES.

Vibrational Raman mode calculation. To identify the vibrational modes associated with the observed wave packets, DFT calculations of vibrational normal modes in the room temperature tetragonal $(14 / \mathrm{mcm})$ phase of $\mathrm{MAPbI}_{3}$ were performed using periodic boundary conditions (see Methods) ${ }^{42}$. Figure 3 presents the vibrational displacements (red arrows) calculated for the 25.6, 49.3 , and $82.9 \mathrm{~cm}^{-1}$ modes of ground state $\mathrm{MAPbI}_{3}$ perovskite energetically lying in the region of interest. These vibrational modes are mostly composed of $\mathrm{I}-\mathrm{Pb}-\mathrm{I}$ bond bending and stretching motions unlike other higher frequency Raman modes above $\sim 100 \mathrm{~cm}^{-1}$ which involve MA motion ${ }^{42}$. Figure 3 a depicts a large $\mathrm{I}-\mathrm{Pb}-\mathrm{I}$ bending motion at $25.6 \mathrm{~cm}^{-1}$ which can be described as a $\mathrm{B}_{1 \mathrm{~g}}$ mode in $\mathrm{D}_{4 \mathrm{~h}}^{18}$ symmetry group $(14 / \mathrm{mcm})$ space group, while Fig. $3 \mathrm{~b}$ presents another $\mathrm{I}-\mathrm{Pb}-\mathrm{I}$ bending motion in the ab-plane at $49.3 \mathrm{~cm}^{-1}\left(\mathrm{~A}_{1 \mathrm{~g}}\right.$ mode in $\left.\mathrm{D}_{4 \mathrm{~h}}^{18}\right)$. On the other hand, the $82.9 \mathrm{~cm}^{-1}$ mode in Fig. 3(c) displays the $c$-axis $\mathrm{Pb}-\mathrm{I}$ stretching displacement $\left(\mathrm{A}_{1 \mathrm{~g}}\right.$ in $\left.\mathrm{D}_{4 \mathrm{~h}}^{18}\right)$ that effectively modulates the height of the octahedral cell. Based on the reasonable assumption that low frequency skeletal modes are similar in the ground and excited state, these three vibrational modes can be assigned to the experimentally measured wave packet modes at 21,43 , and $75 \mathrm{~cm}^{-1}$, respectively (Fig. 2) and used to depict distortion pathways of the octahedral $\mathrm{PbI}_{6}{ }^{4-}$ structure in the excited-state. Some disagreement between the DFT simulations and experiment is expected for low-frequency vibrational modes (here $\sim 18,9.0$, and $9.0 \%$ for three modes in question) due to the shallow potential energy surface and the harmonic approximation used for analysis. For example, a recent study compared IR absorption and DFT results showing $\sim 20 \%$ deviations in $<100 \mathrm{~cm}$ -1 region ${ }^{43}$. Thus, to reduce the discrepancy, we previously considered several additional effects that need to be taken into account to properly compare calculations with experiments which improved the reliability of our assignments ${ }^{42}$.

Polaron state geometry calculation. To theoretically simulate the structural changes of excited $\mathrm{PbI}_{6}{ }^{4-}$ DFT simulations of finite clusters of $\mathrm{MAPbI}_{3}$ perovskites were performed following procedures previously outlined in our recent work ${ }^{34}$. The subsequent analysis is based on CAM-B3LYP functional (see our previous work $^{34}$ and Methods). Specifically, we obtained geometries of a $\mathrm{MAPbI}_{3}$ neutral cluster and a cluster with an extra electron mimicking negative polaron generation. The computationally determined ground state structure was previously verified by $\mathrm{XRD}$ experiments, and is therefore more reliable compared to the geometry of charged states ${ }^{34}$. Figure 4 shows the structurally optimized clusters of the simulated neutral and negative polaron states of $\mathrm{MAPbI}_{3}$ perovskite. In the polaron structure, the methylammonium counterions reorient their electric dipoles (indicated by red arrows) toward the center $\mathrm{Pb}$ atom to minimize the free energy of the localized electron density in the center $\mathrm{Pb}$ atom $^{34}$. In addition, the $\mathrm{Pb}-\mathrm{I}$ bonds, especially in the central $\mathrm{PbI}_{6}{ }^{4-}$ octahedra, also experience substantial structural changes, when an electron is added. Figure $4 \mathrm{c}$ compares the central $\mathrm{PbI}_{6}{ }^{4-}$ octahedron structure of the neutral $\left(\mathbf{S}_{\text {neu }}\right.$, red sphere) and the 

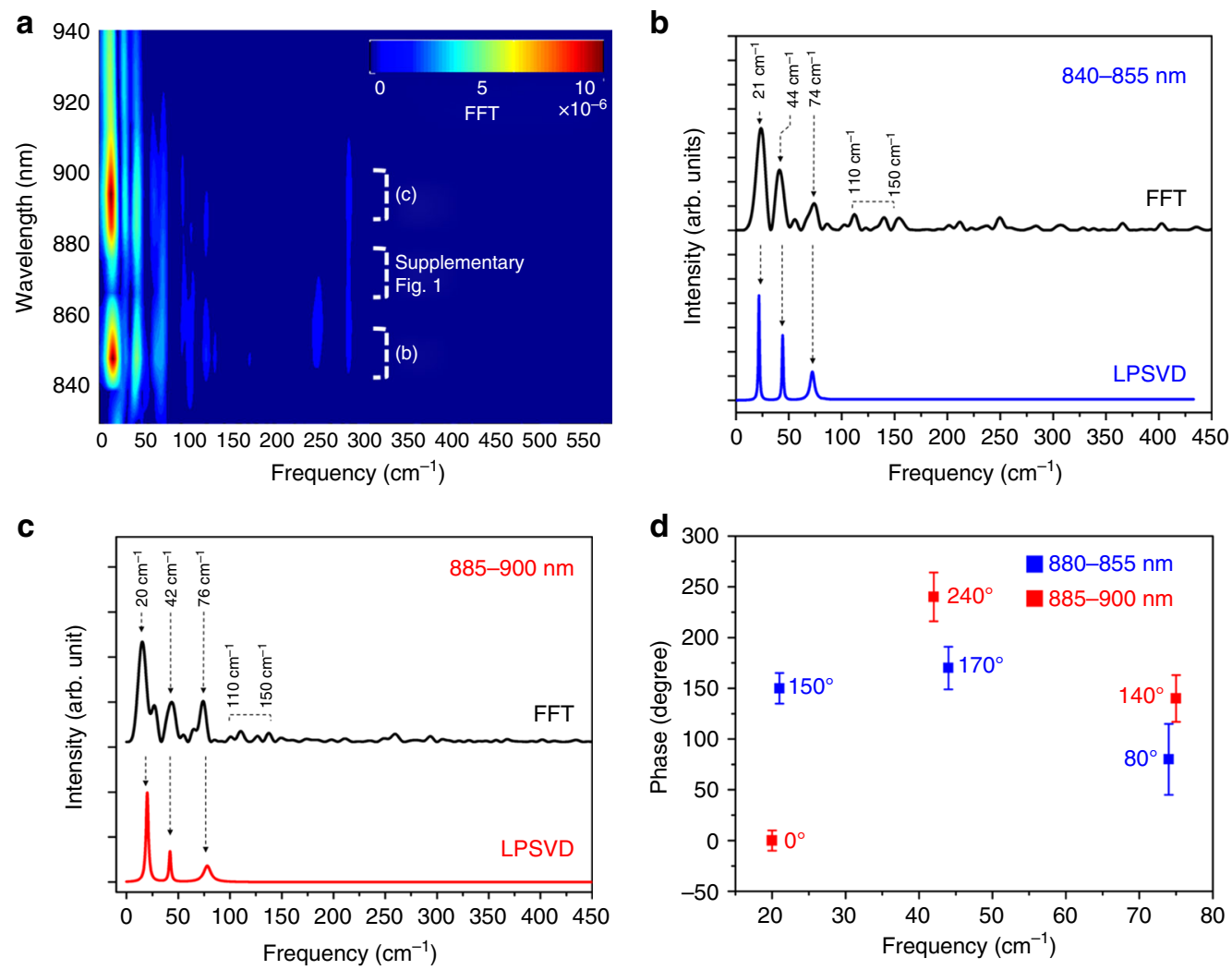

Fig. 2 FFT spectra of Fig. 1. a Contour plot of FFT spectra measured in the entire probe pulse range for MAPbl 3 perovskite (830-940 nm). b, c FFT and LPSVD spectra measured at $840-855 \mathrm{~nm}$ (blue) and at $885-900 \mathrm{~nm}$ (red). d Display of the relative phases of the three principal LPSVD peaks fit on the red and blue sides of the band
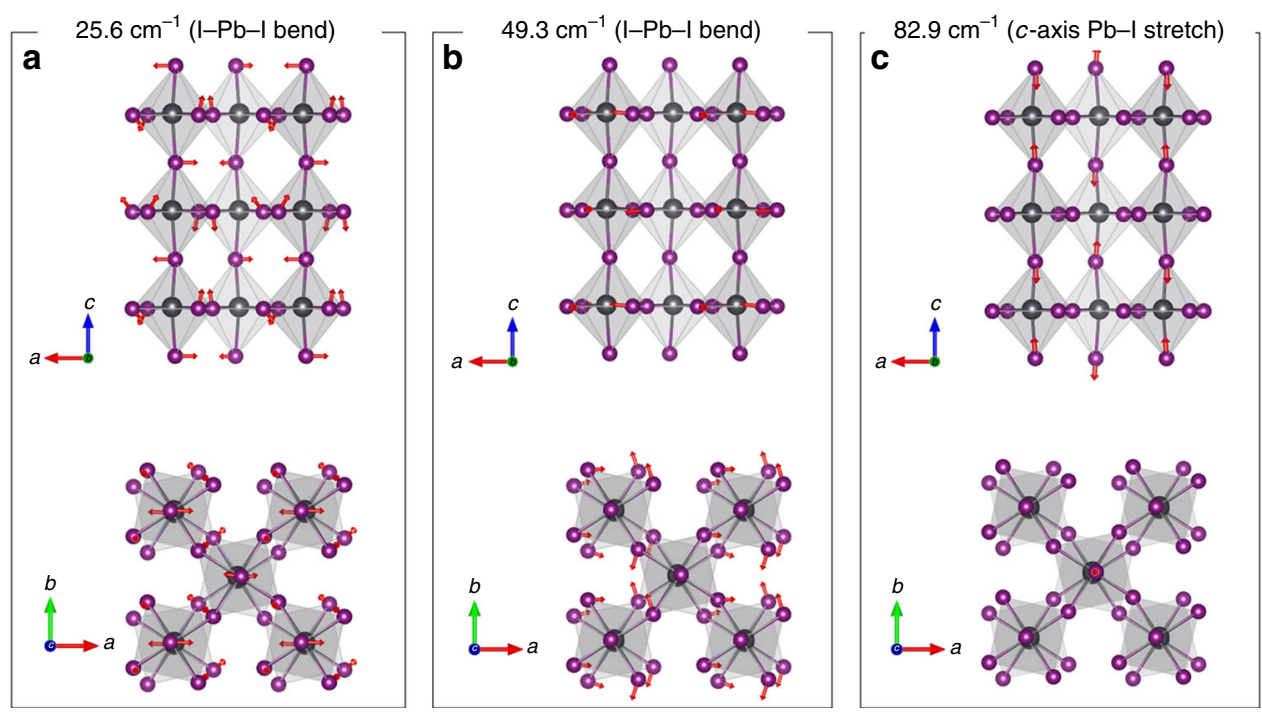

Fig. 3 DFT calculated vibrational modes of $\mathrm{MAPbl}_{3}$. a Skeletal I-Pb-I bending motion of the $\mathrm{Pbl}_{6}{ }^{4-}$ octahedron at $25.6 \mathrm{~cm}^{-1}$. $\mathbf{b} \mathrm{I}-\mathrm{Pb}-\mathrm{I}$ bending motion on the $a b$-plane at $49.3 \mathrm{~cm}^{-1}$. c Pb-I c-axis stretching motion at $82.0 \mathrm{~cm}^{-1}$. Red arrows on the atoms indicate the direction and relative amplitude of displacements in the given vibrational modes

polaron $\left(\mathbf{S}_{\mathrm{pol}}\right.$, blue sphere). The most unique structural difference between $\mathbf{S}_{\text {neu }}$ and $\mathbf{S}_{\mathrm{pol}}$ is the octahedron height along $z$-axis; the polaron $\mathrm{PbI}_{6}{ }^{4-}$ has a $\mathrm{Pb}-\mathrm{I}(5)$ distance of $4.2 \AA$ which is considerable longer than $3.3 \AA$ for the neutral one. In addition, the neutral $\mathrm{PbI}_{6}{ }^{4-}$ has a nearly right angle $\left(89^{\circ}\right)$ for the $\mathrm{I}(5)-\mathrm{Pb}-\mathrm{I}(4)$ bond, while in the polaron the bond angle is $84^{\circ}$. Thus, the polaron has a distorted and bent octahedral structure, while the neutral geometry forms a nearly regular octahedron. These differences are indicated by the displacement vectors (green arrows) in Fig. 4c, which are composed of bending and stretching motions of the I-Pb-I bond.

The structural differences predicted for the excited polaron state geometry should be related to the vibrational relaxation toward the charge state minimum that occurs after Franck-Condon (FC) excitation of the neutral form. Thus, the measured vibrational modes oscillating in the ESA should be the 
a

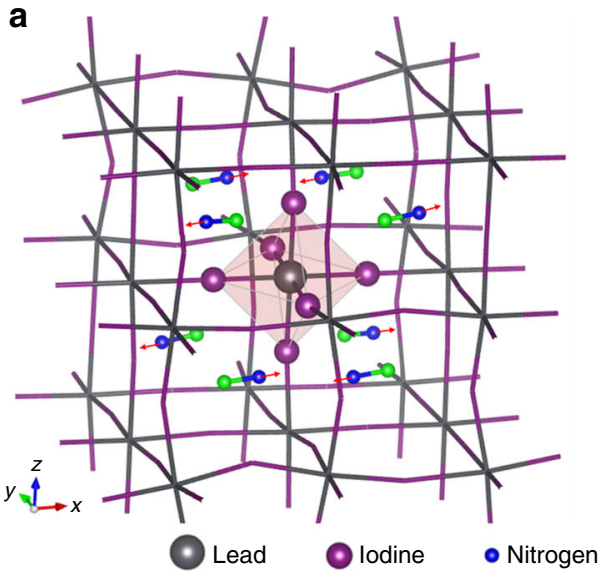

b

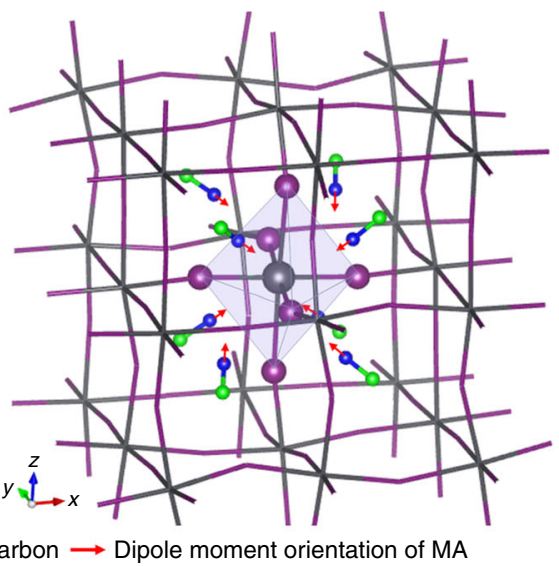

C

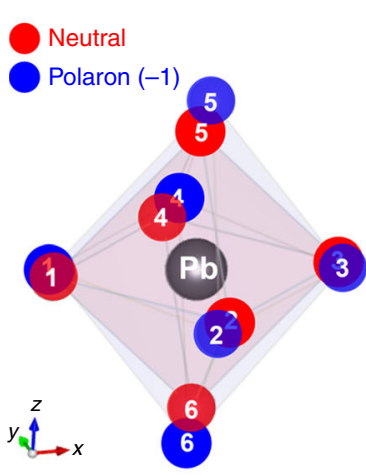

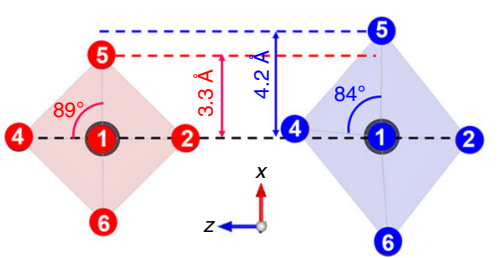

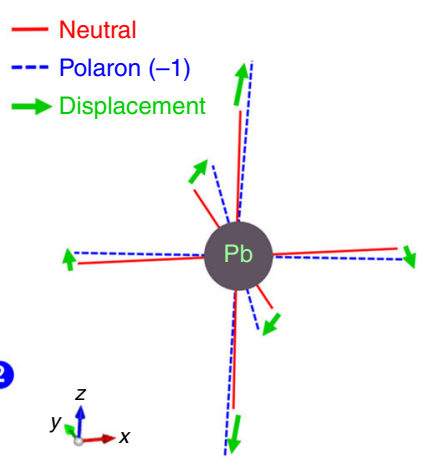

Fig. 4 Theoretically predicted cluster structures of $\mathrm{MAPbl}_{3}$ perovskite. a Neutral cluster. b Polaron (-1) cluster. c Various perspective comparisons of the neutral (red sphere) and polaron (blue sphere) $\mathrm{Pbl}_{6}{ }^{4-}$ octahedral structures centered in the clusters. The structural comparison shows that the polaron state geometry is more bent and elongated than the neutral, indicated by displacement vectors (green arrows)

modes that trigger the nuclear reorganization that forms the excited polaron state. In order to test this hypothesis, we quantitatively investigated the relation between the experimental excited-state vibrational oscillation intensities (Figs. 1-3) and the polaron structural predictions (Fig. 4).

Experimental and theoretical displacement analysis. The key part of our analysis is relating the oscillatory signals in the ESA in Figs. 1 and 2 with the displacement of the excited state of perovskite along the observed vibrational modes. In the timedependent picture of Raman intensities, these excited state oscillations are precisely the nuclear motions that give rise to the resonance Raman scattering process. Since the Raman scattering cross-sections $\left(\sigma_{R}\right)$ are approximately proportional to $\Delta^{2} \omega^{2}$ in the short-time limit, where $\Delta$ and $\omega$ are the ground-to-excited state displacement of the potential minima and the vibration frequency, respectively ${ }^{44-46}$, the relative displacement ratio for each Raman peak can be determined. Using this relationship, the relative displacement ratio $\left(\Delta_{\operatorname{Exp}}\right)$ is found to be $\pm 4.3, \pm 1.4$, and \pm 1.0 for the $\sim 20, \sim 45$, and $\sim 75 \mathrm{~cm}^{-1}$ modes in the impulsive stimulated Raman spectra (ISRS) in Fig. 2. The absolute phases cannot be determined from the experimental data alone because of the $\Delta^{2}$ relationship. The $\Delta_{\text {Exp }}$ result shows that the I-Pb-I bending motion at $\sim 20 \mathrm{~cm}^{-1}$ is the most highly displaced vibrational normal mode, and thus, the most responsible for the excited-state PES deformation that we have probed in the TA. The other bending and stretching modes provide lower displacement contributions to the excited-state PES. Thus, using the $\Delta_{\text {Exp }}$, we can experimentally estimate a polaron state structure
$\left(\mathbf{S}_{\mathrm{Exp}}\right)$ given by

$$
\mathbf{S}_{\text {Exp }}=\mathbf{S}_{\text {neu }}+p\left(\Delta_{\text {Exp }} \cdot \mathbf{c}_{\text {neu }}\right)
$$

where $p$ is a proportional factor and $\mathbf{C}_{\text {neu }}$ is a matrix containing the Cartesian-coordinate vibration eigenvectors of each atom of the neutral state. Notably, Eq. (1) does not rely on the less accurate theoretical $\mathbf{S}_{\text {pol }}$ for excited state structure. Figure $5 \mathrm{a}$ presents the comparison of $\mathbf{S}_{\text {neu }}$ (red), $\mathbf{S}_{\text {pol }}$ (blue), and $\mathbf{S}_{\mathrm{Exp}}$ (green) with $p=1.6$ from the best nonlinear square fit toward $\mathbf{S}_{\mathrm{pol}}$ $\left(\chi_{r}^{2} \sim 1.43\right)$ when $4.3,1.4$, and -1.0 are used for $\Delta_{\text {Exp }}$. We determined the phases of the $\Delta_{\text {Exp }}$ from inspection of the theoretical polaron geometry in Fig. $4 \mathrm{~b}$. It is evident that the green atoms of the reconstructed $\mathbf{S}_{\mathrm{Exp}}$ move toward the blue $\left(\mathbf{S}_{\mathrm{pol}}\right)$ from the red $\left(S_{\text {neu }}\right)$ locations, thus affirming the successful simulation of the theoretically observed polaron state structure using the relative displacement ratio, $\Delta_{\text {Exp }}$.

A complementary approach for analyzing these geometric changes is to perform a normal mode projection from the $\mathbf{S}_{\text {neu }}$ to $S_{\text {pol }}$ by relying solely on theoretical predictions ${ }^{47}$. The theoretical displacement $\left(\Delta_{\text {Theo }}\right)$ is given by

$$
\Delta_{\text {Theo }}=\mathbf{l}_{\text {neu }}^{-1} \mathbf{c}_{\text {neu }}^{T}\left(\mathbf{S}_{\text {pol }}-S_{\text {neu }}\right),
$$

where $\mathbf{l}$ is a diagonal matrix containing $l_{i i}=\left(\frac{\hbar}{2 \pi \nu_{i}}\right)^{\frac{1}{2}}$, and $\nu_{i}$ is the $i^{\text {th }}$ vibration frequency. $\Delta_{\text {Theo }}$ are determined to be $+2.8,+0.3$, and -5.1 for the 20,45 , and $75 \mathrm{~cm}^{-1}$ vibration modes, respectively. In addition, the excited-state reorganization energy $(\lambda)$ is calculated to be $145 \mathrm{meV}\left(\sim 1170 \mathrm{~cm}^{-1}\right)$ given by $\lambda=\frac{h}{2} \sum \nu_{i} \Delta_{i}^{2}$, where $h$ is Planck constant. This value is very 
a

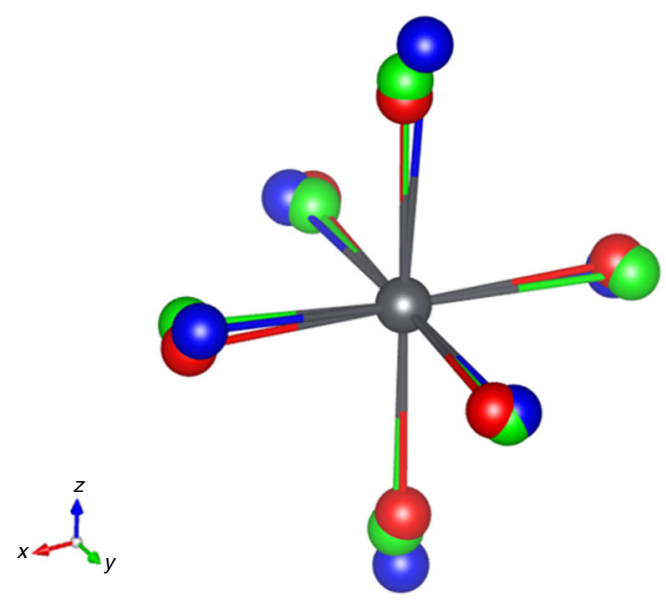

Neutral $\odot$ Polaron (-1) $\odot$ Reconstructed with $\Delta_{\text {EXP }}$

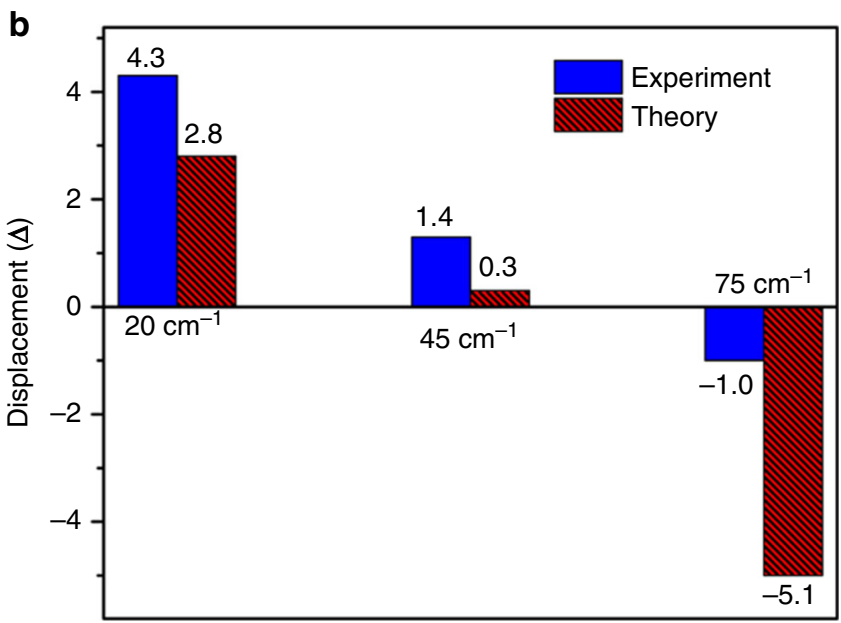

Raman mode

Fig. 5 Structural comparisons between experiment and theory. a Reconstructed $\mathrm{Pbl}_{6}{ }^{4-}$ octahedral structure (green) from $\Delta_{\operatorname{Exp}}$ and Eq. (1) compared with the theoretically predicted neutral (red) and polaron (blue) state structures. b Displacement comparisons between the experiment $\left(\Delta_{\text {Exp }}\right)$ and theory $\left(\Delta_{\text {Theo }}\right)$

consistent with the band gap stabilization energy $(\sim 160 \mathrm{meV})$ found in a periodic boundary condition (PBC) DFT calculation ${ }^{34}$, indicating that our observed vibration modes represent the majority of polaron stabilization dynamics.

Figure 5 shows that the combined $\Delta_{\text {Exp }}$ and $\Delta_{\text {Theo }}$ results are in good qualitative agreement; both experimental and theoretical displacements show that the $20 \mathrm{~cm}^{-1}$ displacement is larger than the $45 \mathrm{~cm}^{-1}$ displacement, both confirming that the whole octahedral I-Pb-I bending motion at $\sim 20 \mathrm{~cm}^{-1}$ provides strong displacements toward the polaron state. However, the displacement -5.1 of $75 \mathrm{~cm}^{-1}$ mode of $\Delta_{\text {Theo }}$ is larger than the experimentally observed $\Delta_{\text {Exp }}$ at $75 \mathrm{~cm}^{-1}$. This inconsistency principally results from the different heights of $S_{\text {pol }}(4.2 \AA)$ and $\mathbf{S}_{\text {Exp }}(3.6 \AA)$ which result from an overestimation of $S_{\text {pol }}$ due to the use of limited atom numbers in the calculation and an underestimation of $\mathbf{S}_{\exp }$ due to the limited time-resolution of the experiment which damps the oscillatory signal strength for the higher frequency mode. However, both our methods (Eqs. (1) and (2)) present a consistent picture about the elevated octahedral heights from $\mathbf{S}_{\text {neu }}$. Thus, the theoretically predicted polaron geometry is nicely supported by the experimentally resolved vibrational displacements, showing that $\mathrm{I}-\mathrm{Pb}-\mathrm{I}$ bending and stretching motions are the key displacements stabilizing polaron formation.

\section{Discussion}

Our nuclear wave packet results and theoretical simulations on $\mathrm{MAPbI}_{3}$ perovskite show how the polaron state is coherently generated on the displaced PES. Electronic excitation above the band gap $(\sim 1.65 \mathrm{eV})$ of $\mathrm{MAPbI}_{3}$ produces the coherences as determined in the current study, ultimately leading to the separation of an initial excitation into free charges in view of weak excitonic effects in $\mathrm{MAPbI}_{3}{ }^{48,49}$. The observed dynamic vibrational excitations facilitate this relaxation toward different structures, where a localized charge is further stabilized through the structural deformation of the Pb-I framework (Fig. 4) and the MA electric dipole reorientation; this is the polaron state hypothesis $^{34}$. The reorientation time $(\tau)$ of the solvated MA was measured to be $\sim 150$ fs by using optical Kerr-gate spectroscopy and ultrafast IR spectroscopy; this time is most relevant for ultrafast coherent solvation in solutions ${ }^{33,50}$. In this context, we have found small FFT peaks at $\sim 150 \mathrm{~cm}^{-1}$ (see Figs. 1 and 2) which are likely due to MA's libration motion ${ }^{42}$. However, the MA coherence is found to be much smaller than the $\mathrm{Pb}-\mathrm{I}$ coherences, showing that the $\mathrm{Pb}-\mathrm{I}$ motions present the principal FFT peaks at low frequencies. This suggests that the MA reorientational motion is likely to be a more continuous (incoherent) solvent-like movement stabilizing the localized polaron ${ }^{50}$, while the measured $\mathrm{Pb}-\mathrm{I}$ vibrations are coherent processes which trigger the Jahn-Teller distortion.

$\mathrm{Pb}$ and I atoms are covalently bonded to form the sturdy perovskite framework. However, once more charge character is introduced (especially, into the $\mathrm{Pb}$ atom), this $\mathrm{Pb}-\mathrm{I}$ framework becomes more ionic ${ }^{51}$ and the transition is facilitated by $\mathrm{Pb}-\mathrm{I}$ structural distortions shown as the bending and elongations of the $\mathrm{Pb}-\mathrm{I}$ bonds mirrored in our theoretical studies (Fig. 4). Our key finding is that this distortion is effectively performed by $\mathrm{Pb}-\mathrm{I}$ coherent vibrational motions over a few picosecond time scale. Interestingly, both experiment and theory agree that the $21 \mathrm{~cm}^{-1}$ mode (I-Pb-I bending) should be the principal nuclear distortion motion for polaron formation, in good agreement with the strong electron-phonon coupling at $0.65 \mathrm{THz}\left(22 \mathrm{~cm}^{-1}\right)$ probed by femtosecond terahertz emission ${ }^{51}$ and the light-induced I-Pb-I angle displacement measured by electron scattering 52 .

Figure 6 displays a schematic two-dimensional PES diagram for the polaron state formation. The PES for the neutral and polaron states have different potential minima which are $\mathbf{S}_{\text {neu }}(\mathbf{0}$, 0) and $S_{\text {pol }}\left(\Delta_{\text {Str }}, \Delta_{\text {Bend }}\right)$, respectively. The geometry changes are determined predominantly by an $\mathrm{I}-\mathrm{Pb}-\mathrm{I}$ bending normal mode at $21 \mathrm{~cm}^{-1}$ (Fig. 3a) and an I-Pb-I $c$-axis stretching normal mode at $75 \mathrm{~cm}^{-1}$ (Fig. 3c). After excitation to the Franck-Condon state, $\mathbf{S}_{\mathrm{FC}}(\mathbf{0}, \mathbf{0})$, the displacements cause the excited-state nuclear wave packets to evolve along these multi-dimensional I-Pb-I vibration coordinates coherently propagating excited-state population toward the polaron state minima $S_{\text {pol }}\left(\Delta_{\text {Str }}, \Delta_{\text {Bend }}\right)$ in the first few picoseconds. Thus, we suggest that coherent polaron formation dynamics is linked to the efficient charge-separation of $\mathrm{MAPbI}_{3}$ perovskite by directing the phase-space evolution to the polaron state faster than competing relaxation mechanisms. Other reports have suggested the subsequent dynamics of charge carriers as large polarons, where limited charge-carrier mobility was linked to coupling to LO phonon branches with frequency of $88 \mathrm{~cm}^{-153,54}$.

In summary, we performed femtosecond TA and DFT simulations to probe the excited-state dynamics of $\mathrm{MAPbI}_{3}$ and found evidence for coherent skeletal vibration dynamics that lead to 


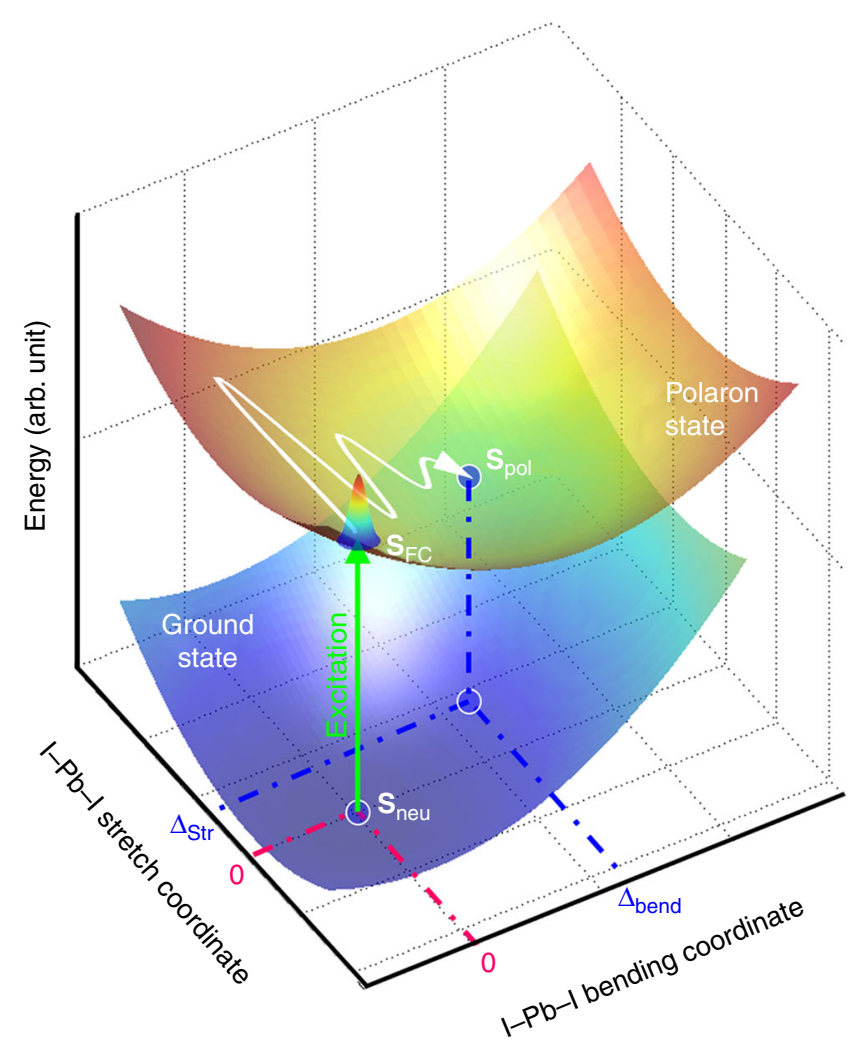

Fig. 6 Excited-state potential energy surface diagram. The diagram displays the atomically displaced $\left(\Delta_{S t r}, \Delta_{\text {Bend }}\right)$ polaron state surface relative to the ground state $(0,0)$ neutral surface after excitation. A coherent nuclear wave packet oscillates along the I-Pb-I stretching coordinate and propagates toward $\Delta_{\text {Bend }}$ along the I-Pb-I bending coordinate. $\mathbf{S}_{\mathrm{FC}}, \mathbf{S}_{\text {neur }}$ and $\mathbf{S}_{\text {pol }}$ are relative structures of Franck-Condon state, neutral, and polaron states

formation of a polaron state underscoring charge separation. The low frequency wave packet modes are observed principally for the $\mathrm{Pb}-\mathrm{I}$ bending and stretching vibrations resulting from the different geometry of the polaron state compared to the neutral state. We analyzed the experimental and theoretical displacements and confirmed that the theoretical negative polaron state structure is supported by the experimental Raman intensity analysis results. Thus, the distorted octahedral geometry of the $\mathrm{PbI}_{6}{ }^{4-}$ polaron state structure is characterized, and its initial formation dynamics are demonstrated to be dominated by the coherent nuclear wave packet motion. The high efficiency of $\mathrm{MAPbI}_{3}$ perovskite solar cells may be yet another example of the importance of vibrational coherence in efficient photochemical dynamics $^{55-57}$

\section{Methods}

Sample preparation. Samples were prepared by dissolving $0.76 \mathrm{mmol} \mathrm{PbI}_{2}$

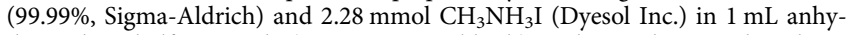
drous dimethylformamide (DMF, Sigma-Aldrich), and stirred overnight. The prepared solution was spun on $\mathrm{O}_{2}$-plasma treated glass substrates at $1500 \mathrm{rpm}$ for $60 \mathrm{~s}$, and then annealed at $120^{\circ} \mathrm{C}$ for $60 \mathrm{~min}$ in a $\mathrm{N}_{2}$-filled glovebox. Poly(methyl methacrylate) (PMMA) was coated on the fresh $\mathrm{MAPbI}_{3}$ layer to protect it from the atmosphere. We have checked powder X-ray diffraction (XRD) measured by using a Bruker AXS D8 Advance diffractometer with a $\mathrm{Cu}$ Ka source. (Supplementary Fig. 2)

Pump-probe spectroscopy. A home-built Kerr lens mode-locked Ti:sapphire oscillator ( $20 \mathrm{fs}$ FWHM, $5.3 \mathrm{~nJ} /$ pulse, $91 \mathrm{MHz})$ seeded a Ti:sapphire regenerative amplifier (B.M. Industries, Alpha $1000 \mathrm{US}, 991 \mathrm{~Hz}, 70 \mathrm{fs}, 780 \mu \mathrm{J} / \mathrm{pulse}, \lambda \max =790$ $\mathrm{nm}$ ) pumped by a Q-switched Nd:YLF (B.M. Industries, 621-D). The fundamental at $790 \mathrm{~nm}$ was used to generate visible pump pule ( $\sim 35 \mathrm{fs}$ FWHM, $150 \mathrm{~nJ} / \mathrm{pulse}$, $\lambda \max =560 \mathrm{~nm}$ ) by a non-collinear optical parametric amplifier (NOPA). A negatively chirped mirror pair (Layertec $\mathrm{GmbH}$ ) was used to compress the pump pulse. A small portion of the fundamental is focused onto a $3 \mathrm{~mm}$ thick sapphire plate to generate a broadband continuum probe pulse ( $10 \mathrm{fs}$ FWHM, $\sim 3 \mathrm{~nJ} /$ pulse). The probe pulse was temporally compressed in the $830-940 \mathrm{~nm}$ region with a pair of BK7 dispersion prisms (Supplementary Fig. 3). The cross-correlation function was measured to be a Gaussian function with $\sim 44$ fs FWHM by using the optical Kerr effect in cyclohexane (Supplementary Fig. 4). The power of the pump pulse was reduced to $\sim 6 \mathrm{~nJ}$ to avoid thermal and photochemical damage to the sample. A custom-made $\mathrm{N}_{2}$ gas-flow chamber was used to prevent humidity-degradation of the $\mathrm{MAPbI}_{3}$ sample. A monochromator (Instruments SA, HR320), a lasersynchronized CCD (Princeton Instruments, PIXIS), and an optical chopper (Newport, 3501) were used to measure the optical density (OD) differences in every pump/probe cycle (Supplementary Fig. 3). Python and Matlab software were used to control the experimental instruments and analyze data, respectively.

Calculation of vibration frequencies. First-principles density functional theory (DFT) calculations were performed using VASP 58,59 . Our VASP calculations use the generalized gradient approximation $(\mathrm{GGA})^{59}$, and projected augmented-wave pseudopotentials ${ }^{60,61}$. Structural and electronic properties were computed using a plane-wave energy cut-off of $500 \mathrm{eV}$ and a $4 \times 4 \times 4$ Monkhorst-Pack k-point grid $^{62}$, including spin-orbit interactions (SOI). Lattice constants and internal atomic positions were relaxed starting from the experimental structure, without symmetry constrains, until forces were smaller than $1 \mathrm{meV} / \AA \AA$. Phonon eigenfrequencies and eigenvectors were calculated in the harmonic approximation, neglecting SOI. Our phonon calculations use density functional perturbation theory and a finer $6 \times 6 \times 6 \mathrm{k}$-point grid. Selected phonon modes and electronic orbitals were plotted using VESTA. The room temperature tetragonal $(\mathrm{I} / \mathrm{mcm})$ phase of $\left(\mathrm{CH}_{3} \mathrm{NH}_{3}\right) \mathrm{PbI}_{3}$ is simulated in a $\sqrt{2} \times \sqrt{2} \times 2$ perovskite unit cell. The tetragonal structure consists of a $\mathrm{PbI}_{6}$ octahedral rotation around the $z$-axis $\left(\mathrm{a}^{0} \mathrm{a}^{0} \mathrm{c}\right.$ in Glazer notation) and an antiparallel orientation of the $\mathrm{CH}_{3} \mathrm{NH}_{3}$ (MA) molecules $^{42,63,64}$.

Optimization of polaron structure. All calculations of HOIP isolated clusters were performed using the CAM-B3LYP functional combined with the LANL2dz (for Pb and $\mathrm{I}$ ) and $6-31 \mathrm{G}^{*}$ (for $\mathrm{N}, \mathrm{C}$, and $\mathrm{H}$ ) basis sets using Gaussian 09 software package ${ }^{65}$. In addition, we include a polar solvent $(\varepsilon=78)$ via the conductor-like polarizable continuum model ${ }^{66}$.

Several considerations were taken into account when designing the isolated HOIP structures. We started with the low temperature cubic lattice parameters that were derived from variable temperature powder $x$-ray diffraction experiments ${ }^{67}$. In order to minimize dangling bonds, the cluster was terminated on all sides with MAI. Equations 1 and 2 ensure that the system is overall neutral

$$
6 n_{\mathrm{Pb}}=2 n_{I_{1}}+n_{I_{2}}
$$

$$
2 n_{\mathrm{Pb}}+n_{\mathrm{MA}}=n_{I_{1}}+n_{I_{2}}
$$

Here $n_{\mathrm{Pb}}, n_{\mathrm{MA}}$, and $n_{\mathrm{I}}$ are the number of $\mathrm{Pb}, \mathrm{MA}$, and I atoms, respectively. $I_{1}$ corresponds to an atom on the surface and is only bonded to one $\mathrm{Pb}$ atom, while $I_{2}$ corresponds to an I atom that is bonded to two $\mathrm{Pb}$ atoms. The cluster needs to be large enough to include at least $8 \mathrm{MA}$ cations surrounding the central $\mathrm{Pb}$ atom. To fulfill all conditions, we end up with a cluster having the following stoichiometry: $\mathrm{MA}_{54} \mathrm{~Pb}_{27} \mathrm{I}_{108}\left(I_{1}=I_{2}=54\right)$. A cube fully terminated by MA and I would require 64 MA molecules. In order to realize charge balance between the ions and maximize symmetry, MA's were removed from the 8 corners and on two opposite edges.

In addition, the polaron geometry in HSE hybrid potential was checked. Supplementary Fig. 5 shows the optimized geometries from CAM-B3LYP (19 to $60 \% \mathrm{HF}$ ) and HSE ( 25 to $0 \% \mathrm{HF}$ ) functionals. The arrows point in the direction the atoms move in the formation of the polaron. This shows that the trend (I-Pb-I bending \& $c$-axis elongation) still holds although the amplitude changes slightly.

Raman mode assignment. Since we measured the excited-state Raman signals originated from the structural displacements $(\Delta)$ between the ground and excited states in $\mathrm{MAPbI}_{3}$, we considered Rashba band splitting and polaron formation for the reason of the excited-state displacements. The former one is related to IR-active vibration modes, because the splitting is promoted with directional structural strains. Meanwhile the latter, the polaronic $\mathrm{PbI}_{6}{ }^{4-}$ is rather induced by symmetric structural changes from the neutral $\mathrm{PbI}_{6}{ }^{4-}$ of the ground state, because a negative charge $(-1)$ is localized in $6 \mathrm{p}$ orbital of the center $\mathrm{Pb}$. Thus, this polaronic structure shows the $\mathrm{Pb}$-centered symmetric distortion shown in Fig. 4. Based on this idea, our Raman mode assignments have been done with the octahedral $\mathrm{PbI}_{6}{ }^{4-}$ Raman modes in the $\mathrm{D}_{4 \mathrm{~h}}$ Raman mode pool ${ }^{43}$. Thus, the $\mathrm{Pb}$-centered Raman modes are easily discriminated from other IR-active and $\mathrm{Pb}$-moving Raman modes (Supplementary Fig. 6). 
Data availability. All data supporting the findings of this study are available from the corresponding author upon reasonable request.

Received: 13 December 2017 Accepted: 1 June 2018

Published online: 28 June 2018

\section{References}

1. Green, M. A. et al. Solar cell efficiency tables (version 50). Prog. Photo.: Res. Appl. 25, 668-676 (2017).

2. Wang, H. \& Kim, D. H. Perovskite-based photodetectors: materials and devices. Chem. Soc. Rev. 46, 5204-5236 (2017).

3. Shin, S. S. et al. Tailoring of electron-collecting oxide nanoparticulate layer for flexible perovskite solar cells. J. Phys. Chem. Lett. 7, 1845-1851 (2016).

4. Seo, G. et al. Microscopic analysis of inherent void passivation in perovskite solar cells. ACS Energy Lett. 2, 1705-1710 (2017).

5. Kim, Y. C. et al. Beneficial effects of $\mathrm{PbI}_{2}$ incorporated in organo-lead halide perovskite solar cells. Adv. Energy Mater. 6, 1502104 (2016).

6. Lai, M. et al. Structural, optical, and electrical properties of phase-controlled cesium lead iodide nanowires. Nano Res 10, 1107-1114 (2017).

7. Tsai, H. et al. High-efficiency two-dimensional Ruddlesden-Popper perovskite solar cells. Nature 536, 312-316 (2016).

8. Pedesseau, L. et al. Advances and promises of layered halide hybrid perovskite semiconductors. ACS Nano 10, 9776-9786 (2016).

9. Buriak, J. M. et al. Virtual issue on metal-halide perovskite nanocrystals-a bright future for optoelectronics. Chem. Mater. 29, 8915-8917 (2017).

10. Xing, J. et al. High-efficiency light-emitting diodes of organometal halide perovskite amorphous nanoparticles. ACS Nano 10, 6623-6630 (2016)

11. Yang, S., Fu, W., Zhang, Z., Chen, H. \& Li, C.-Z. Recent advances in perovskite solar cells: efficiency, stability and lead-free perovskite. J. Mater. Chem. A 5, 11462-11482 (2017).

12. Yang, W. S. et al. Iodide management in formamidinium-lead-halide-based perovskite layers for efficient solar cells. Science 356, 1376-1379 (2017).

13. Jeon, N. J. et al. Solvent engineering for high-performance inorganic-organic hybrid perovskite solar cells. Nat. Mater. 13, 897-903 (2014)

14. Yang, W. S. et al. High-performance photovoltaic perovskite layers fabricated through intramolecular exchange. Science 348, 1234-1237 (2015).

15. Park, N.-G. Methodologies for high efficiency perovskite solar cells. Nano Converg. 3, 15 (2016).

16. Heo, $\mathrm{S}$. et al. Deep level trapped defect analysis in $\mathrm{CH}_{3} \mathrm{NH}_{3} \mathrm{PbI}_{3}$ perovskite solar cells by deep level transient spectroscopy. Energy Environ. Sci. 10, 1128-1133 (2017).

17. Gélvez-Rueda, M. C. et al. Effect of cation rotation on charge dynamics in hybrid lead halide perovskites. J. Phys. Chem. C. 120, 16577-16585 (2016)

18. Leblebici, S. Y. et al. Facet-dependent photovoltaic efficiency variations in single grains of hybrid halide perovskite. Nat. Energy 1, 16093 (2016).

19. Thu Ha, Do. T. et al. Optical study on intrinsic exciton states in high-quality $\mathrm{CH}_{3} \mathrm{NH}_{3} \mathrm{PbBr}_{3}$ single crystals. Phys. Rev. B 96, 075308 (2017).

20. Venkatesan, S. et al. Tailoring nucleation and grain growth by changing the precursor phase ratio for efficient organic lead halide perovskite optoelectronic devices. J. Mater. Chem. C 5, 10114-10121 (2017)

21. Jonathan, D. M. Grain boundaries in CdTe thin film solar cells: a review. Semicond. Sci. Technol. 31, 093001 (2016).

22. Rißland, S. \& Breitenstein, O. Evaluation of recombination velocities of grain boundaries measured by high resolution lock-in thermography. Energy Procedia 38, 161-166 (2013).

23. Yang, J., Siempelkamp, B. D., Liu, D. \& Kelly, T. L. Investigation of $\mathrm{CH}_{3} \mathrm{NH}_{3} \mathrm{PbI}_{3}$ degradation rates and mechanisms in controlled humidity environments using in situ techniques. ACS Nano 9, 1955-1963 (2015).

24. Zhu, X. Y. \& Podzorov, V. Charge carriers in hybrid organic-inorganic lead halide perovskites might be protected as large polarons. J. Phys. Chem. Lett. 6, 4758-4761 (2015).

25. Kang, B. \& Biswas, K. Shallow trapping vs. deep polarons in a hybrid lead halide perovskite, $\mathrm{CH}_{3} \mathrm{NH}_{3} \mathrm{PbI}_{3}$. Phys. Chem. Chem. Phys. 19, 27184-27190 (2017).

26. Stranks, S. D. et al. Electron-hole diffusion lengths exceeding 1 micrometer in an organometal trihalide perovskite absorber. Science 342, 341-344 (2013).

27. Oga, H., Saeki, A., Ogomi, Y., Hayase, S. \& Seki, S. Improved understanding of the electronic and energetic landscapes of perovskite solar cells: high local charge carrier mobility, reduced recombination, and extremely shallow traps. J. Am. Chem. Soc. 136, 13818-13825 (2014).

28. Wehrenfennig, C., Liu, M., Snaith, H. J., Johnston, M. B. \& Herz, L. M. Charge-carrier dynamics in vapour-deposited films of the organolead halide perovskite $\mathrm{CH}_{3} \mathrm{NH}_{3} \mathrm{PbI}_{3-x} \mathrm{Cl}_{x}$. Energy Environ. Sci. 7, 2269-2275 (2014).

29. Miyata, K. et al. Large polarons in lead halide perovskites. Sci. Adv. 3, e1701217 (2017).
30. Ivanovska, T. et al. Long-lived photoinduced polarons in organohalide perovskites. J. Phys. Chem. Lett. 8, 3081-3086 (2017).

31. Welch, E., Scolfaro, L. \& Zakhidov, A. Density functional theory+U modeling of polarons in organohalide lead perovskites. AIP Adv. 6, 125037 (2016).

32. Niesner, D. et al. Persistent energetic electrons in methylammonium lead iodide perovskite thin films. J. Am. Chem. Soc. 138, 15717-15726 (2016).

33. Zhu, H. M. et al. Screening in crystalline liquids protects energetic carriers in hybrid perovskites. Science 353, 1409-1413 (2016).

34. Neukirch, A. J. et al. Polaron stabilization by cooperative lattice distortion and cation rotations in hybrid perovskite materials. Nano. Lett. 16, 3809-3816 (2016).

35. Lee, G., Kim, J., Kim, S. Y., Kim, D. E. \& Joo, T. Vibrational spectrum of an excited state and Huang-Rhys factors by coherent wave packets in timeresolved fluorescence spectroscopy. Chemphyschem 18, 670-676 (2017).

36. Hoffman, D. P. \& Mathies, R. A. Femtosecond stimulated Raman exposes the role of vibrational coherence in condensed-phase photoreactivity. Acc. Chem. Res. 49, 616-625 (2016)

37. Park, M., Im, D., Rhee, Y. H. \& Joo, T. Coherent and homogeneous intramolecular charge-transfer dynamics of 1-tert-Butyl-6-cyano-1,2,3,4tetrahydroquinoline (NTC6), a rigid analogue of DMABN. J. Phys. Chem. A 118, 5125-5134 (2014).

38. Dietze, D. R. \& Mathies, R. A. Femtosecond stimulated Raman spectroscopy. Chemphyschem 17, 1224-1251 (2016).

39. Wang, H., Valkunas, L., Cao, T., Whittaker-Brooks, L. \& Fleming, G. R. Coulomb screening and coherent phonon in methylammonium lead iodide perovskites. J. Phys. Chem. Lett. 7, 3284-3289 (2016).

40. Pollard, W. T., Lee, S. Y. \& Mathies, R. A. Wave packet theory of dynamic absorption-spectra in femtosecond pump-probe experiments. J. Chem. Phys. 92, 4012-4029 (1990).

41. Musser, A. J. et al. Evidence for conical intersection dynamics mediating ultrafast singlet exciton fission. Nat. Phys. 11, 352-357 (2015).

42. Park, M. et al. Critical role of methylammonium librational motion in methylammonium lead iodide $\left(\mathrm{CH}_{3} \mathrm{NH}_{3} \mathrm{PbI}_{3}\right)$ perovskite photochemistry. Nano. Lett. 17, 4151-4157 (2017).

43. Pérez-Osorio, M. A. et al. Vibrational properties of the organic-inorganic halide perovskite $\mathrm{CH}_{3} \mathrm{NH}_{3} \mathrm{PbI}_{3}$ from theory and experiment: factor group analysis, first-principles calculations, and low-temperature infrared spectra. J. Phys. Chem. C 119, 25703-25718 (2015).

44. Hoffman, D. P., Ellis, S. R. \& Mathies, R. A. Low frequency resonant impulsive Raman modes reveal inversion mechanism for azobenzene. J. Phys. Chem. A 117, 11472-11478 (2013).

45. Gong, K., Kelley, D. F. \& Kelley, A. M. Resonance Raman spectroscopy and electron-phonon coupling in zinc selenide quantum dots. J. Phys. Chem. C. 120, 29533-29539 (2016)

46. Grenland, J. J., Lin, C., Gong, K., Kelley, D. F. \& Kelley, A. M. Resonance Raman investigation of the interaction between aromatic dithiocarbamate ligands and CdSe quantum dots. J. Phys. Chem. C. 121, 7056-7061 (2017)

47. Reimers, J. R. A practical method for the use of curvilinear coordinates in calculations of normal-mode-projected displacements and Duschinsky rotation matrices for large molecules. J. Chem. Phys. 115, 9103-9109 (2001).

48. Soufiani, A. M. et al. Impact of microstructure on the electron-hole interaction in lead halide perovskites. Energy Environ. Sci. 10, 1358-1366 (2017).

49. March, S. A. et al. Four-wave mixing in perovskite photovoltaic materials reveals long dephasing times and weaker many-body interactions than GaAs. ACS Photonics 4, 1515-1521 (2017).

50. Bakulin, A. A. et al. Real-time observation of organic cation reorientation in methylammonium lead iodide perovskites. J. Phys. Chem. Lett. 6, 3663-3669 (2015).

51. Burak, G. et al. Terahertz emission from hybrid perovskites driven by ultrafast charge separation and strong electron-phonon coupling. Adv. Mater. 30, 1704737 (2018).

52. Wu, X. X. et al. Light-induced picosecond rotational disordering of the inorganic sublattice in hybrid perovskites. Sci. Adv. 3, 7 (2017).

53. Wright, A. D. et al. Electron-phonon coupling in hybrid lead halide perovskites. Nat. Commun. 7, 11755 (2016)

54. Herz, L. M. Charge-carrier mobilities in metal halide perovskites: fundamental mechanisms and limits. ACS Energy Lett. 2, 1539-1548 (2017).

55. Eames, C. et al. Ionic transport in hybrid lead iodide perovskite solar cells. Nat. Commun. 6, 7497 (2015).

56. Mathies, R. A. PHOTOCHEMISTRY: a coherent picture of vision. Nat. Chem. 7, 945-947 (2015)

57. Fang, C., Frontiera, R. R., Tran, R. \& Mathies, R. A. Mapping GFP structure evolution during proton transfer with femtosecond Raman spectroscopy. Nature 462, 200-204 (2009).

58. Kresse, G. \& Furthmüller, J. Efficient iterative schemes for ab inito totalenergy calculations using a plane-wave basis set. Phys. Rev. B 54, 11169-11186 (1996). 
59. Perdew, J. P., Burke, K. \& Ernzerhof, M. Generalized gradient approximation made simple. Phys. Rev. Lett. 77, 3865-3868 (1996).

60. Blöchl, P. E. Projector augmented-wave method. Phys. Rev. B 50, 17953-17979 (1994).

61. Kresse, G. \& Joubert, D. From ultrasoft pseudopotentials to the projector augmented-wave method. Phys. Rev. B 59, 1758-1775 (1999).

62. Monkhorst, H. J. \& Pack, J. D. Special points for Brillouin-zone integrations. Phys. Rev. B 13, 5188-5192 (1976).

63. Kawamura, Y., Mashiyama, H. \& Hasebe, K. Structural study on cubictetragonal transition of $\mathrm{CH}_{3} \mathrm{NH}_{3} \mathrm{PbI}_{3}$. J. Phys. Soc. Jpn. 71, 1694-1697 (2002).

64. Quarti, C., Mosconi, E. \& De Angelis, F. Interplay of orientational order and electronic structure in methylammonium lead iodide: Implications for solar cell operation. Chem. Mater. 26, 6557-6569 (2014).

65. Frisch, M. J. et al. Gaussian 09 Revision B.01 (Gaussian Inc. Wallingford CT, 2009).

66. Barone, V. \& Cossi, M. Quantum calculation of molecular energies and energy gradients in solution by a conductor solvent model. J. Phys. Chem. A 102, 1995-2001 (1998).

67. Baikie, T. et al. Synthesis and crystal chemistry of the hybrid perovskite $\left(\mathrm{CH}_{3} \mathrm{NH}_{3}\right) \mathrm{PbI}_{3}$ for solid-state sensitised solar cell applications. J. Mater. Chem. A 1, 5628-5641 (2013).

\section{Acknowledgements}

The spectroscopic and theoretical works at University of California, Berkeley by M.P. was supported by the Mathies Royalty Fund. The work at Los Alamos National Laboratory (LANL) by A.J.N and S.T. was supported by the LANL LDRD program and was done in part at Center for Nonlinear Studies (CNLS) and the Center for Integrated Nanotechnologies CINT), a U.S. Department of Energy and Office of Basic Energy Sciences user facility, at LANL. This research used resources provided by the LANL Institutional Computing Program. LANL is operated by Los Alamos National Security, LLC, for the National Nuclear Security Administration of the U.S. Department of Energy under contract DE-AC52-06NA25396. S.E.R.-L. and J.B.N. acknowledge support from the Molecular Foundry (supported by the Office of Science, Office of Basic Energy Sciences, of the U.S. Department of Energy), and the Laboratory Directed Research and Development Program at the Lawrence Berkeley National Laboratory under Contract No. DEAC02-05CH11231. The material preparation work by M.L. was supported by the U.S. Department of Energy, Office of Science, Office of Basic Energy Sciences, Materials Sciences and Engineering Division, under Contract No. DE-AC02-05-CH11231 within the Physical Chemistry of Inorganic Nanostructures Program (KC3103). M.L. thanks Suzhou Industrial Park for the fellowship support. S.E.R.-L. thanks Dirección General de
Investigación at Universidad Andres Bello for support under Proyecto Interno Regular DI-21-18/“REG”.

\section{Author contributions}

M.P. and R.A.M. conceived the project; M.P. collected and analyzed the TA data with the theoretical projection methods with contribution from S.R.E and D.D.; A.J.N undertook the polaron structure optimization with contributions from S.T.; S.E.R.-L. undertook the vibration frequency calculation with contribution from J.B.N.; M.L. prepared the sample and collected additional data with contribution from P.Y.; M.P., A.J.N., S.E.R.-L., S.T., and R.A.M. wrote the manuscript with contributions and advice from all others.

\section{Additional information}

Supplementary Information accompanies this paper at https://doi.org/10.1038/s41467018-04946-7.

Competing interests: The authors declare no competing interests.

Reprints and permission information is available online at http://npg.nature.com/ reprintsandpermissions/

Publisher's note: Springer Nature remains neutral with regard to jurisdictional claims in published maps and institutional affiliations.

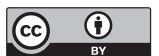

Open Access This article is licensed under a Creative Commons Attribution 4.0 International License, which permits use, sharing, adaptation, distribution and reproduction in any medium or format, as long as you give appropriate credit to the original author(s) and the source, provide a link to the Creative Commons license, and indicate if changes were made. The images or other third party material in this article are included in the article's Creative Commons license, unless indicated otherwise in a credit line to the material. If material is not included in the article's Creative Commons license and your intended use is not permitted by statutory regulation or exceeds the permitted use, you will need to obtain permission directly from the copyright holder. To view a copy of this license, visit http://creativecommons.org/ licenses/by/4.0/.

(c) The Author(s) 2018 